\title{
Mobilité à des fins d'apprentissage :Un modèle conceptuel et un dispositif de médiation pour des processus réussis de mobilité
}

Marisa Cavalli et Mirjam Egli Cuenat

\section{(2) OpenEdition}

1 Journals

Édition électronique

URL : http://journals.openedition.org/rdlc/6904

DOI : $10.4000 /$ rdlc.6904

ISSN : 1958-5772

Éditeur

ACEDLE

Référence électronique

Marisa Cavalli et Mirjam Egli Cuenat, « Mobilité à des fins d'apprentissage :Un modèle conceptuel et un dispositif de médiation pour des processus réussis de mobilité », Recherches en didactique des langues et des cultures [En ligne], 16-2 | 2019, mis en ligne le, consulté le 20 novembre 2019. URL http://journals.openedition.org/rdlc/6904; DOI : 10.4000/rdlc.6904

\section{(c) $(1) \odot \Theta$}

Recherches en didactique des langues et des cultures is licensed under a Creative Commons AttributionNonCommercial-NoDerivatives 4.0 International License 
Mobilité à des fins d'apprentissage :

Un modèle conceptuel et un dispositif de médiation pour des processus réussis de mobilité

Marisa Cavalli

Ex Institut Régional de Recherche Educative pour le Val d'Aoste (IRRE-VDA)

Consultante auprès du Centre Européen des Langues Vivantes (CELV) du Conseil de l'Europe

mrcavalli@alice.it

https://delanguesetdautre.com

Mirjam Egli Cuenat

Haute école pédagogique de la Haute école spécialisée du nord-ouest de la Suisse (FHNW)

Chaire de la didactique du français et de ses disciplines (Ecole primaire)

mirjam.egli@fhnw.ch

https://www.fhnw.ch/de/personen/mirjam-egli

\section{Résumé en français}

Le texte aborde la mobilité à des fins d'apprentissage, organisée dans un cadre éducatif et proposée aux apprenants voire aux enseignants en formation. Un modèle conceptuel, élaboré dans le cadre des travaux de l'Unité des Politiques Linguistiques du Conseil de l'Europe dans lequel la médiation tient une place centrale en tant qu'élément facilitateur de divers processus (de projection, d'inclusion et de focalisation), permettra de caractériser un projet de mobilité vu sous un angle formateur. Les méthodologies et les moyens pédagogiques auxquelles il s'agit de former les apprenants en mobilité s'inspireront notamment d'outils conceptuels développés par le projet PluriMobil réalisé au Centre Européen des Langues Vivantes du Conseil de l'Europe. Ces méthodologies et moyens, envisagés comme autant d'actions et/ou de dispositifs de médiation, encouragent la réflexivité, tant au niveau linguistique que (inter)culturel.

\section{Mots-Clés}

Mobilité à des fins d'apprentissage, médiation, processus de projection, processus d'inclusion, processus de focalisation, réflexivité

\section{Résumé en anglais}

The paper deepens mobility for learning purposes, organised in an educational framework and offered to learners or even teachers in training. A conceptual model, developed as part of the work of the Council of Europe's Language Policy Unit, in which mediation plays a central role as a facilitating element in various processes (of projection, inclusion and focus), allows to describe a mobility project from an educational perspective. The methodologies and the pedagogical means for training learners in mobility can be based on a variety conceptual tools such as those developed by the PluriMobil project at the European Centre for Modern Languages of the Council of Europe. These methodologies and means, envisaged as actions and/or mediation devices, foster reflexivity, both at the linguistic and the (inter)cultural level.

\section{Keywords}

Mobility for learning purposes, mediation, projection process, inclusion process, focus process, reflexivity 


\section{Introduction}

Notre réflexion sur les «mobilités ${ }^{1}$ se pose d'emblée comme interdisciplinaire car elle investit divers champs :

- une conception renouvelée de la formation de l'individu permettant de prendre en compte les transformations et les nouvelles contraintes imposées par notre époque presque trente ans après les premières élaborations du Cadre Européen Commun de Référence (CECR);

- une certaine idée du rôle des mobilités dans le processus d'apprentissage, de développement et de changement ;

- une représentation du concept de médiation en relation aussi bien avec le processus de mobilité, qu'avec l'entrée dans de nouveaux groupes ou communautés et l'élaboration constructive des perceptions altéritaires nécessaire pour un apprentissage réellement réussi.

Notre texte s'organisera comme suit : l'attention sera d'abord portée sur une définition du type de mobilité dont nous traiterons - la mobilité à des fins d'apprentissage - et un panorama rapide des différents types et formes de cette mobilité organisés dans un cadre éducatif et proposés aux apprenants aux différents niveaux d'apprentissage voire aux enseignants en formation initiale ou continue (partie 2). Nous présenterons ensuite le modèle conceptuel général dans lequel s'insère notre réflexion, élaboré dans le cadre des travaux de l'ancienne Unité des Politiques Linguistiques du Conseil de l'Europe (Coste \& Cavalli, 2015) qui nous permettra de caractériser de façon spécifique un projet de mobilité, vu sous un angle global et largement formateur. Ce modèle a été conçu afin de rendre compte des déplacements notionnels qui ont pu avoir lieu au cours des années qui ont séparé la première conceptualisation du CECR (début des années 90), sa publication en 2001 et les temps actuels. Il entend tenir compte des changements sociétaux intervenus entre-temps au niveau conceptuel aussi bien que, pour le cas présent, dans les pratiques didactiques liées à la mobilité. Comme les développements suivants vont le montrer, la médiation y tient une place centrale en tant qu'élément facilitateur de divers processus (partie 3). Par la suite (partie 4) seront précisés, sur la base d'autres publications récentes, les méthodologies auxquelles il s'agit de former les sujets en mobilité et les moyens pédagogiques destinés à encourager les expériences de mobilité et à développer des compétences utiles. Ces méthodologies et moyens pédagogiques seront caractérisés, en référence au modèle présenté, comme autant de formes et/ou de dispositifs de médiation qui peuvent être mis à profit des processus de mobilité à des fins d'apprentissages. Cette partie sera illustrée plus particulièrement à partir des outils conceptuels développés dans le cadre du projet PluriMobil (Egli Cuenat et al., 2015). Ce faisant, nous partons du constat émis par plusieurs chercheurs dans le domaine selon lesquels une mobilité à des fins d'apprentissage qui ne se double pas d'une formation suivie risque de produire des résultats pauvres ou superficiels, voire de renforcer les préconçus (voir p.ex. Jackson, 2012).

\section{La mobilité à des fins d'apprentissage}

\subsection{Définition}

Dans la littérature, on trouve une multitude de concepts plus ou moins équivalents : mobilité à des fins d'apprentissage, échanges linguistiques et culturels, échanges internationaux, éducation internationale, séjour linguistique, stage, étude, séjour à l'étranger. Cela se différencie encore

\footnotetext{
${ }^{1}$ Nous assumons ici le pluriel d'autant plus que si, d'un côté, la tâche impartie par les organisateurs de la journée NEQ a été celle de prendre en compte aussi bien les mobilités physiques que les mobilités symboliques, d'un autre, et de façon plus pertinente par rapport à notre réflexion, un projet de mobilité à des fins d'apprentissage (cf. infra) n'est à notre avis réussi que dans la mesure où les mobilités physiques s'accompagnent de mobilités symboliques se situant à divers niveaux (conscientisation, réflexivité, ouverture culturelle ...). Dans la suite du texte, le singulier sera adopté pour rendre compte d'une forme particulière de mobilité, la mobilité à des fins d'apprentissage.
} 
suivant les langues : en anglais, par exemple, les termes utilisés sont Learning mobility, Education abroad, Study abroad, Residence abroad, International education.

La plupart de ces termes comportent des limitations, puisqu'ils se focalisent sur un aspect de la mobilité qui peut en exclure d'autres. Nous privilégierons le concept «mobilité à des fins d'apprentissage ", car il désigne la finalité première de ce genre de mobilité qui peut se réaliser sous différentes formes. Nous en adoptons la définition suivante, tirée du manuel PluriMobil, qui se base pour partie sur le Livre Vert de la Commission européenne (2009) :

expériences éducatives faites dans de nouveaux environnements et visant de façon explicite à améliorer les compétences plurilingues, interculturelles (et professionnelles) des apprenants. La durée typique des activités d'apprentissage en mobilité est de quelques jours ou semaines pour les apprenants d'écoles primaires et secondaires, à des périodes plus longues comme un ou deux semestres d'université dans le cas de jeunes adultes étudiant dans des instituts ou universités de formation des enseignants.

La mobilité peut désigner non seulement des contacts transfrontaliers, mais aussi la coopération intra-nationale, spécialement quand il s'agit de pays multilingues (Egli Cuenat et al., 2015, PluriMobil Manuel : 15).

Cette définition large inclut également la mobilité virtuelle au moyen des technologies de l'information et de la communication (TIC). La mobilité à des fins d'apprentissage est pleinement reconnue comme pouvant contribuer au développement de compétences langagières/plurilingues, interculturelles (de communication) (Coste, Moore \& Zarate, 1997 ; 2009) et à la formation globale de l'individu (cf. p.ex. Jackson, 2012 ; Gohard-Radenkovic, 2007 ; Murphy-Lejeune, 2004, entre autres).

\subsection{Types et formes d'apprentissage en mobilité}

L'apprentissage en mobilité impliquant un déplacement physique peut prendre différentes formes, avoir différentes durées, viser différentes destinations, prévoir différentes formes de logements etc. Il en va de même pour la mobilité virtuelle qui grâce à des outils tels que des blogs, des vidéoconférences, des plates-formes d'apprentissage en ligne, etc., permet de coopérer avec des étudiants d'origines culturelles et linguistiques diverses sans changer d'environnement physique d'apprentissage (Livre vert, 2009, 4 et ss.).

Les initiatives destinées à organiser et promouvoir la mobilité à des fins d'apprentissage sont désormais très nombreuses et elles sont promues par des instances aux plus divers échelons institutionnels et administratifs (Commission européenne, États, collectivités territoriales, associations et organisations non gouvernementales, institutions de formation à tous les niveaux des systèmes éducatifs ...).

Certaines sont internationales et peuvent concerner un continent entier (cf. Erasmus + ) et au-delà (cf. Erasmus mundus), d'autres relèvent d'accords bi-nationaux (par ex. : les échanges francoallemands ${ }^{2}$ ), d'autres encore d'initiatives nationales (par ex. l'agence nationale suisse MOVETIA ${ }^{3}$ ) ou locales (les établissements scolaires par ex.). Les programmes de mobilité sont désormais foison

\footnotetext{
${ }^{2}$ Pour les enseignants du primaire http://eduscol.education.fr/cid46951/echange-franco-allemand-d-enseignants-du-1er-degre-20182019.html; pour la jeunesse https://www.ofaj.org (consultés le 06/09/19).

${ }^{3}$ https://www.movetia.ch/fr/(consulté le 06/09/19).
} 
surtout s'ils concernent les apprenants de langues pour lesquels le secteur privé multiplie les propositions $^{4}$.

\section{Un modèle conceptuel et ses mises à l'épreuve}

Dans le but de situer la mobilité à des fins d'apprentissage dans une réflexion plus large, de l'encadrer différemment et afin de caractériser d'un point de vue formateur toutes les mesures d'accompagnement nécessaires à sa réussite, nous nous servirons d'un modèle conceptuel élaboré dans le cadre des travaux de l'ancienne Unité des politiques linguistiques du Conseil de l'Europe. Ce modèle s'est construit autour de trois notions que le CECR, au moment de sa première élaboration (début des années 90) et de sa publication (2001), n'a que partiellement - ou, pour certaines, pas du tout - prises en compte : mobilité, communauté et altérité. Il faut préciser que ces trois notions ont été assumées dans le modèle avec un sens beaucoup plus large, presque métaphorique, que leur acception dans le CECR où elles n'apparaissent pas - ou bien ne sont pas thématisées - en tant que telles. Elles se différencient également des notions ayant cours dans d'autres cadres théoriques actuels de réflexion. Précisons aussi que certaines d'entre elles sont loin d'être neutres, surtout dans certains contextes nationaux. Elles ont été retenues car elles permettent mieux que d'autres d'asseoir une réflexion générale pouvant aisément s'adapter à - et rendre compte de - différents contextes où différents acteurs sont impliqués. La notion de médiation s'articule fortement et différemment à ces trois notions.

Ce modèle avait pour ambition (modeste) d'être utile pour analyser différentes situations actuelles où les compétences langagières et culturelles ont un rôle important à jouer pour l'avenir des acteurs : qu'il s'agisse de la situation d'apprentissage d'un élève en milieu scolaire, de celle du migrant dans la société d'accueil, de celle de l'étudiant en mobilité voire du futur enseignant en formation, etc. Il a été pour cela mis à l'épreuve de différents contextes :

- le domaine de l'éducation - au moment de la publication de l'étude pour le Conseil de l'Europe (Coste \& Cavalli, 2015) - de façon à mettre en relation les évolutions sociétales en cours et les évolutions envisageables au niveau scolaire ${ }^{5}$;

- le milieu carcéral ${ }^{6}$ pour tracer le développement d'un projet personnel ;

- la situation des élèves issus de la migration de première et de deuxième génération (Coste \& Cavalli, 2019);

- la réflexion interdisciplinaire sur les éléments transversaux de la médiation dans divers domaines disciplinaires (Coste \& Cavalli, sous presse).

Dans le cadre de ce papier, le modèle sera donc utilisé pour caractériser la mobilité à des fins d'apprentissage comme un type de mobilité parmi d'autres, pour cerner ses potentiels ainsi que pour mettre en évidence la nécessité d'actes de médiation pour en assurer un réel bénéfice.

\subsection{Des notions à articuler}

Le modèle conceptuel peut être caractérisé comme suit ${ }^{7}$ :

\footnotetext{
${ }^{4}$ https://www.cairn.info/revue-cahiers-de-1-action-2015-1-page-94.htm voir Les dispositifs de mobilité pour les jeunes, des opportunités pour agir (consulté le 06/09/19).

${ }^{5}$ A remarquer qu'une première version du texte (Coste \& Cavalli, 2014a, non publié) prévoyait une tentative d'application du modèle au cas des migrants adultes.

${ }^{6}$ A l'occasion du Convegno internazionale "Il sistema penitenziario e i detenuti stranieri: problematiche, risorse e prospettive", organisé par Antonella Benucci dans le cadre du Progetto RiUscire auprès de l'Università per Stranieri di Siena les 26 et 27 mai 2017 où une communication sur "Lingue e culture al servizio dell'inclusione sociale e dello sviluppo professionale" a été présentée par Marisa Cavalli.

${ }^{7}$ Chaque partie de la définition est introduite par une citation de Coste \& Cavalli (2018 : 245-6) entre guillemets et en italique.
} 
1. «Le sujet apprenant, l'acteur social apprend, se construit, se transforme par des processus de mobilité ».

" Mobilité » est à concevoir ici en tant que " déplacement dans un espace ou un monde différent (" espace » et " monde » étant ici entendus au sens large), effectué par un acteur ou un groupe social. Sont ainsi couvertes les situations spécifiées par des adjectivations diverses, telles que : mobilité géographique, mobilité sociale, mobilité économique, mobilité migratoire, mobilité professionnelle ; mais aussi : mobilité universitaire, mobilité scolaire » (Coste \& Cavalli, 2014b : 104). La mobilité concrète constitue, en même temps, une image métaphorique pour la mobilité symbolique. Et par mobilité symbolique - notion souvent évoquée, mais rarement définie dans les nombreuses publications que nous avons consultées - nous entendons deux types de processus : d'un côté, les acquis en termes de " capital culturel ", de " pouvoir symbolique ", de " mise en pouvoir " qui vont faciliter à l'acteur social une inclusion étendue et aisée dans les groupes sociaux auxquels il désire prendre part ; d'un autre côté, les déplacements cognitifs, représentationnels ainsi que le développement personnel qui devraient donner lieu à l'acquisition de la " compétence symbolique » (Kramsch, 2009 : emplacement 4911 et ss., éd. Electronique, notre traduction). Pour Kramsch, la compétence symbolique est « une capacité à la fois théorique et pratique, qui découle de la nécessité pour le sujet de trouver des positions appropriées dans et entre les langues en cause ». Cette compétence qui définit « le sujet multilingue » inclut :

- une capacité à comprendre la valeur symbolique des formes symboliques et les différentes mémoires culturelles évoquées par les différents systèmes symboliques.

- une capacité à s'appuyer sur la diversité sémiotique qu'offre la multiplicité des langues pour recadrer les façons de voir les événements familiers, créer des réalités alternatives et trouver une position de sujet appropriée "entre les langues ", si l'on peut dire.

- une capacité à regarder à la fois la langue et à travers la langue et de comprendre les défis à l'autonomie et à l'intégrité du sujet qui proviennent d'idéologies unitaires et d'une culture totalisante en réseau. (ibidem)

2. «Cette mobilité s'opère à l'intérieur de groupes sociaux ou communautés et par l'introduction dans et la participation à de nouvelles communautés ».

« Communauté » est définie dans les termes suivants : " groupe social (professionnel, académique, religieux, grégaire, sportif ou autre) partageant des caractéristiques ou des intérêts communs et perçu ou se percevant comme à certains égards distincts d'autres groupes à l'intérieur d'une société donnée ou d'un ensemble social plus vaste » (Coste \& Cavalli, 2014b : 106). Elle permet de prendre en compte des groupements, des collectifs ou collectivités, y compris, pour le cas actuel, en raison des connotations ayant à voir avec la relation interculturelle. Donc, la notion de " communauté » aussi doit être comprise au sens large, cela pouvant aller de la classe de mathématiques ou de toute autre discipline à l'école à l'association de volontariat, au groupe sportif, au cours de danse etc. Par rapport au CECR, l'introduction de cette notion empêche une confrontation trop directe entre individu et société et rend compte, de façon plus réaliste, moins vague, des processus de socialisation tels qu'ils se réalisent au quotidien. Il a donc été jugé utile d'avoir recours à cette notion malgré ses connotations culturelles parfois problématiques dans certains contextes.

3. «C'est par la mobilité et l'introduction dans de nouvelles communautés que l'acteur social fait l'expérience de formes d'altérité, relatives à sa propre perception ». 
L' " altérité ", telle que conçue dans le modèle, n'est pas une essence en soi : la notion ne relève donc pas, loin de là, d'une vision essentialisante. Elle est plutôt envisagée comme le résultat de la perception du sujet ou d'un processus de construction sociale; altérité se définit en tant que " caractère de ce qui est autre ", mais avec deux déplacements majeurs par rapport à la plupart des usages circulants : cet « autre » ou ce « nouveau » (cf. le " savoir aborder du nouveau » à propos du « savoir apprendre » selon certaines versions intermédiaires du CECR non publiées ${ }^{8}$ ) est le fruit de la perception individuelle (mais qui peut être socialement construite ou induite et incorporée) et ne se limite pas à un « autrui » humain, pouvant être aussi bien un individu, un groupe, une culture qu'une connaissance à acquérir, une œuvre à découvrir.

4. «La mobilité elle-même, l'introduction et la participation à de nouvelles communautés, la rencontre de l'altérité perçue supposent des capacités d'adaptation culturelle et linguistique et exigent/permettent la mise en place et/ou le développement de compétences et connaissances d'ordre linguistique et culturel pour partie nouvelles ».

L'apprentissage se réaliserait donc à travers un processus de mobilité avec l'entrée dans de nouveaux groupes dont le sujet entend faire partie et à l'intérieur desquels il rencontre des normes, des codes, des connaissances et des pratiques autres, qu'il peut percevoir comme plus ou moins distants et nouveaux pour lui. L'appropriation de ces derniers, indispensables à l'inclusion du sujet, requiert de sa part le déploiement de ressources (linguistiques et culturelles) « déjà là » et l'acquisition de nouveaux moyens.

5. «L'adaptation culturelle et linguistique ne va pas de soi et il importe que diverses modalités de médiation' facilitent pour l'acteur social sa mobilité, son inclusion dans les communautés, son appréhension de l'altérité. Dans tous les cas, l'action de médiation tend, dans sa définition la plus générale, à réduire la distance entre deux pôles distants ou en tension ».

Les pôles peuvent être des instances sociales (acteur social individuel, groupe, institution ...) mais aussi objectif à atteindre, connaissance à acquérir, discipline scolaire à apprendre etc. La tension peut relever aussi bien du conflit, que de la dissymétrie ou de la dénivellation entre les deux pôles quant à l'information, aux connaissances, aux pouvoirs, aux droits et devoirs respectifs ${ }^{10}$. La tension peut être de signe positif enclenchant un mouvement vers l'individu, le groupe, la connaissance etc. ou de signe négatif quand la distance perçue semble trop grande, voire indépassable.

6. «Quelles que soient ses modalités, la médiation est posée soit comme visant l'accès à des informations et connaissances: médiation cognitive, soit comme contribuant à l'interaction, à la qualité de l'échange, à la résolution de conflits: médiation relationnelle.»

La mise en œuvre de la médiation porte soit sur les connaissances soit sur les relations interpersonnelles, bien que, le plus souvent, elle se trouve à prendre en compte en même temps les unes et les autres.

\footnotetext{
${ }^{8}$ D'après Daniel Coste (communication personnelle).

${ }^{9}$ Pour un aperçu historique et interdisciplinaire de la notion de médiation et pour des développements rigoureux autour de l'activité langagière de médiation telle qu'envisagée dans le CECR, cf. Stathopoulou (2015). Cf. aussi sur la médiation culturelle Zarate et al. (2003) ; Lévy \& Zarate (dir.) (2003); Huver \& Lorilleux (dir.) (2018) et Lorilleux \& Huver (dir.) (2018).

${ }^{10}$ En ce sens, la notion de médiation adoptée peut se rapprocher de celle de Six (1990:164) où il est question de liens à nouer ou à renouer ou de conflits à prévenir ou à résoudre et de celle, dans le domaine du droit, de Guillaume-Hofnung (2015 : 69-71) qui envisage que la médiation a à s'exercer entre des différences ou des différends. Ainsi la notion de tension recouvre aussi bien la possibilité que la médiation doive s'appliquer à un éventuel conflit à prévenir ou résoudre qu'à la perspective d'un lien à (re)nouer.
} 
7. «Ces deux formes, cognitive et relationnelle, non exclusives et souvent combinées, passent pour l'essentiel par une activité langagière de médiation (...)».

Quel que soit son objet, la médiation passe toujours par une activité langagière de médiation. C'est en ce sens que le modèle conceptuel proposé offre un cadre élargi pour l'analyse de divers contextes sociaux actuels, pour la définition des besoins que les acteurs y développent en vue de leur pleine inclusion sociale et pour la mise en œuvre d'actions par des instances médiatrices. Dans ce cadre élargi, les activités langagières de médiation offrent un appui important à côté d'autres. Le langage, en effet, est, en même temps, le lieu principal de la médiation (c'est par et dans la parole que les différentes « tensions » entre les pôles en questions se déroulent), son enjeu (toutes les négociations et les successives approximations de sens qui se déroulent entre les pôles en tensions se font par et dans le langage) et le but de toute médiation réussie (c'est par le langage que les dissymétries d'informations ou de connaissances ou encore de pouvoir se rééquilibrent ou que les conflits se résolvent) (Coste \& Cavalli, sous presse).

8. «De plus en plus, les acteurs sociaux sont connectés et membres de réseaux qui peuvent soit renforcer les communautés d'appartenance, soit faciliter l'entrée dans de nouvelles communautés, soit créer de nouveaux collectifs, temporaires ou plus durables ».

Au-delà de l'appartenance à des groupes, l'acteur social fait partie de nos jours de plusieurs réseaux, plus ou moins éphémères, plus ou moins élargis, aux règles plus fluides et à géométrie plus variable, réseaux auxquels le modèle conceptuel fait allusion sans vraiment aborder leur incidence sur le sujet et ses affiliations.

\subsection{Des processus à enclencher dans le cadre de la mobilité à des fins d'apprentissage}

Le modèle s'est enrichi et affiné après sa publication. Sa représentation graphique actuelle est la suivante :

Figure 1 - Le modèle conceptuel d'après Coste \& Cavalli (2015) et Coste (2019).

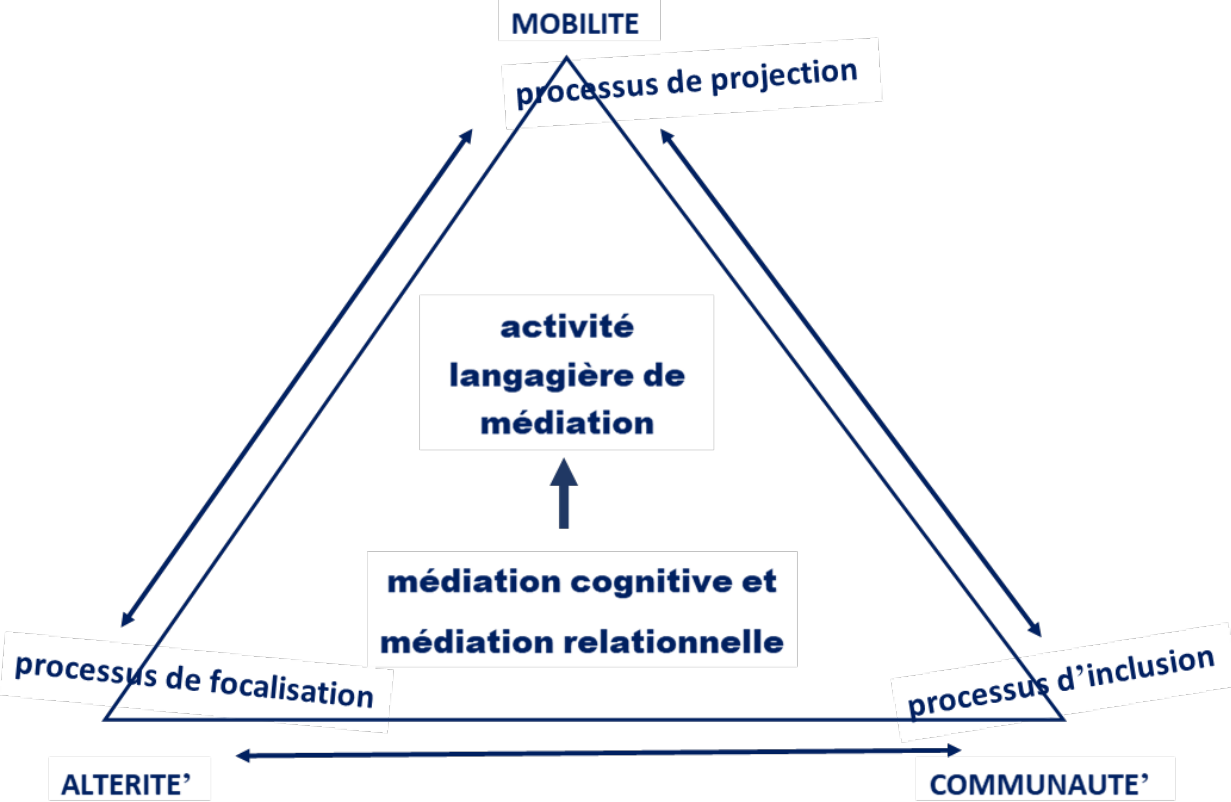


Dans la pratique, le «fonctionnement» de chaque notion donne lieu à des processus (Coste, 2019) que nous allons illustrer pour le cas de la mobilité à des fins d'apprentissage.

Considérons la mise en place voire le déclenchement d'un projet de mobilité à des fins d'apprentissage : le sujet est dans une perspective de projection vers la réalisation de sa mobilité : c'est un moment d'anticipation, d'activation, de préparation de ses déplacements physiques vers de nouvelles communautés / groupes au sein d'un nouvel environnement. Dans le cas de la mobilité virtuelle, ce sera plutôt la rencontre d'un/e partenaire en ligne pour des échanges suivis en tandem ou la participation à un forum international en ligne. Le sujet s'outille et s'arme en faisant des anticipations par rapport aux moyens (linguistiques, culturels, informationnels et autres) qui lui seront utiles pour aborder les défis qui l'attendent et pour profiter à fond de sa future expérience. Il s'agit d'imaginer cette mise en place comme récurrente à chaque fois que dans le nouvel environnement ou espace en ligne le sujet décide d'entrer en contact avec de nouvelles communautés / groupes.

Dans l'enclenchement du processus de mobilité (qu'il s'agisse d'un «voyage », d'un déplacement virtuel ou bien réel), le sujet entre donc dans un(e)/des nouvelle(s) communautés/de nouveau(x) groupe(s). Les processus ici indispensables sont ceux qui facilitent l'inclusion dans ces groupes divers et variés du nouvel environnement dans lesquels le sujet aspire à entrer : dans le cas d'un séjour de mobilité à des fins d'apprentissage, il peut s'agir d'une salle de classe universitaire, d'une salle de classe au lycée, d'une famille d'accueil, d'une entreprise auprès de laquelle on effectue un stage ou bien encore d'un groupe de collègues ou d'un groupe de jeunes sportifs dans lesquels on s'insère, voire des contextes autres où des enquêtes, des interviews, des séances d'observation peuvent avoir lieu.

Au cours de la réalisation de la mobilité et - de façon plus importante, et, en quelque sorte « au second degré », à un niveau plus élevé d'abstraction - au retour au lieu de départ, ce sont les processus de focalisation qui permettent au sujet de constamment vérifier le niveau de distance et de proximité qu'il perçoit en lui par rapport à des attitudes, pratiques, objets, coutumes, événements, discours ... des groupes et communautés qu'il rencontre (ou a rencontrés) dans le pays d'accueil ou en ligne. Ces processus de focalisation amènent le sujet à chercher les ajustements utiles (au niveau aussi bien linguistique qu'informationnel et culturel) pour modifier la perception altéritaire qui pourrait être la sienne.

Ces processus - de projection, d'inclusion, de focalisation - gagnent à être guidés, orientés par l'entremise d'intermédiaires (professionnels - institutions, instruments et outils, dispositifs - ou pas) qui facilitent par leur œuvre médiatrice aussi bien l'intégration de connaissances nouvelles (linguistiques, mais aussi culturelles) que le développement des relations interpersonnelles entre sujets différents, mais aussi entre les sujets eux-mêmes et le nouvel environnement ou la communauté de pratique virtuelle. Cette médiation cognitive et relationnelle passe plus que jamais par l'activité langagière de médiation déployée par les médiateurs (enseignants, formateurs, pairs, ...).

Le maitre-mot du modèle, celui qui le fait fonctionner, qui huile ses mécanismes, est donc « médiation »: qu'elle soit cognitive (apports de connaissances) ou relationnelle (établissement de rapports et de liens, résolution de malentendus ou de conflits), elle passe toujours par l'activité langagière de médiation. C'est cette dernière qui facilite la médiation en tant qu'action visant à réduire la distance entre deux (ou plus de deux) pôles en tension (cf. 3.1.).

Il faut aussi souligner, pour ce qui est de la médiation cognitive, son apport au travail de déconstruction des (pré)conceptions «spontanées », des préconçus, des stéréotypes, de certaines 
représentations sociales et de leurs manifestations langagières figées, circulantes et «socialisées », notamment en relation aux perceptions altéritaires et aux communautés.

Or toutes les recherches sur la mobilité soulignent combien les processus de médiation sont indispensables à la réussite des apprentissages en mobilité, la mobilité en soi pouvant ne pas constituer la garantie que ces apprentissages aient lieu (Paige \& Vande Berg, 2012 ; Roussel \& Gaonac'h, 2017). Le modèle conceptuel, qui vient d'être présenté, nous semble donc bien avoir une pertinence pour des projets de mobilité à des fins d'apprentissage. Il sera montré dans ce qui suit quelles formes concrètes ce travail de médiation peut prendre. Nous sommes là très proches de la médiation vygotskienne (ou encore de l'étayage brunerien), dont l'importance dans les processus de développement et d'autonomisation des apprenants est reprise, entre autres, par Lantolf \& Poehner (2014).

\section{Théories et méthodologies pour le soutien des apprentissages en mobilité}

\subsection{Un champ en pleine expansion}

De nombreuses théories informent le champ d'une pédagogie ou didactique de la mobilité visant à dépasser l'idée que la seule immersion dans des pratiques langagières et culturelles quotidiennes suffit pour déclencher les processus d'apprentissage escomptés (cf., pour un panorama général, Jackson \& Oguro, 2018). Elles se fondent souvent sur des approches théoriques générales qui s'appliquent au domaine éducatif, notamment la conception socioculturelle de l'apprentissage avec la centration sur l'apprenant, la construction sociale de la connaissance, l'étayage, le tutorat, la médiation ... (Vygotsky, 1934/ 1992 ; Bruner, 1978 ; Zarate et al., 2003 ; Lantolf \& Poehner, 2014), la critical theory ou la pédagogie critique (critical language awareness, critical intercultural awareness ...) (Fairclough, 1992 et 2001 ; Guilherme, 2012 ; Dervin, 2016) ; la théorie des communautés de pratique (Lave \& Wenger, 1991) ou encore de l'apprentissage par l'expérience (Kolb,1984).

Parmi les méthodologies concrètes trouvent place les activités réflexives ou méta-réflexives concernant les dimensions (inter)culturelles (Byram, Gribkova \& Starkey, 2002 ; Dervin, 2016) ; les processus d'apprentissage langagier en autonomie dans des situations informelles (Little et al., 2017) ; l'encouragement de la prise de risque dans la communication exolingue telle que décrite par Bange (1992) ; les méthodes (ethnographiques) de recherche / enquête sur le terrain (Blanchet \& Chardenet, 2011); les récits (auto)biographiques, de vie, de parcours de formation, le roman autobiographique ou les lettres (Molinié, 2006); les écrits réflexifs : journaux de bord / d'apprentissage, témoignages, mémoires, compte rendu, blogs ..., les échanges oraux réflexifs (entretiens compréhensifs, discussions en groupe, les tandems), les productions réflexives multimodales (cartes, photos, collages) (Molinié (éd.), 2014) ; l'acquisition de notions scientifiques (anthropologiques, linguistiques, sociologiques), auto-analyse d'écrits personnels, journal des stratégies, discussions ciblées (Dervin, 2016).

La plupart de ces méthodologies permettent de prendre en compte les représentations, les imaginaires, les émotions et les dimensions sensibles impliquées et sollicitées par les expériences de mobilité. Cette prise en compte est tout à fait centrale dans un projet de mobilité, où tous les apprentissages importent et doivent être capitalisés, mais s'agencent, en même temps, autour d'un projet de développement personnel, du début d'un processus que l'on entend durablement et continument transformationnel. Dans le cadre de la mobilité au sein d'une autre communauté linguistique, impliquant un séjour dans un autre pays, il s'agira d'éviter l'écueil d'un déterminisme lié aux appartenances à une culture nationale "essentialisée » (concernant autant «la culture d'origine » que « la culture d'accueil »), sans pour autant tomber dans un relativisme culturel total. 
La décentration est un processus fondamental dans l'apprentissage interculturel quant au développement de la capacité d'adaptation à la diversité culturelle et d'ouverture à tout ce qui est nouveau et autre. Elle touche autant à la prise de distance face à l'expérience habituelle des apprenants qu'à leur conscience d'appartenir à des groupes ou communautés multiples. Elle implique la nécessité de développer une réflexivité, voire une méta-réflexivité sur la manière de percevoir, d'être ou d'agir (Paige \& Vande Berg, 2012 ; Dervin, 2016).

Bien entendu, la prise en compte de ces dimensions sensibles, outre qu'elle requiert une posture éthique de respect et d'accueil de la part des sujets médiateurs, relève davantage de démarches qualitatives que d'approches prétendant à l'objectivité. Pour ces dimensions, en effet, l'objectif de la décentration s'obtient par divers moyens et elle est facilitée par une réflexion non pas en vase clos mais collaborative (qu'elle soit menée en groupe (groupes de discussion), en tandem, avec des pairs ou avec un mentor/ médiateur/facilitateur et qu'elle porte sur des pratiques (linguistiques, culturelles, ...) vécues ou observées. La dimension de l'échange, de la comparaison de points de vue voire d'un guidage critique de la part d'un médiateur/d'une instance médiatrice, permet d'enrichir et de diversifier la réflexion, d'opérer des déplacements cognitifs que l'individu tout seul ne serait probablement pas capable de faire (nous sommes proches ici de la zone de développement proximal de Vygotsky).

\subsection{Encadrement pédagogique des processus de mobilité : l’exemple de PluriMobil}

La littérature de recherche pédagogique montre qu'un soutien focalisé et structuré des phases avant, pendant et après la mobilité est un facteur crucial du succès d'apprentissage des mobilités de tous genres (Kötter, 2013 ; Jackson, 2012 ; Ehrenreich et al., 2008). PluriMobil est un outil destiné à l'encadrement pédagogique des processus de mobilité, basé sur cette structure ternaire. Il a été réalisé auprès du Centre européen pour les langues vivantes du Conseil de l'Europe (CELV) pendant les deux programmes à moyen terme 2008-2011 et 2012-2015. Le produit final comprend cinq séries de plans de leçons adaptés aux différents niveaux scolaires (primaire, secondaire inférieur, secondaire supérieur général et professionnel ainsi que la formation initiale des enseignants), un manuel (Egli Cuenat et al., 2015) et un guide de démarrage rapide qui sont disponibles gratuitement sur le site web du projet (plurimobil.ecml.at, consulté le 06/09/19).

Les matériaux de PluriMobil, en tant que dispositif de médiation pédagogique, s'orientent aux impératifs du terrain de la classe, fournissant une structure simple par scénarios d'apprentissage couvrant les phases avant - pendant-après; chaque plan de leçon est également structuré selon ce principe et contient des propositions pour des objectifs de compétences, contenus et activités pour chaque phase de la mobilité, adaptés au niveau scolaire et à l'âge. Les plans de leçons peuvent être combinés de manière flexible et servir de soutien pour le développement de scénarios accompagnant toute sorte de mobilités. Chaque plan de leçon contient une section sollicitant les apprenants à recueillir les traces d'apprentissage et produits résultant des activités proposées dans un portfolio qui documente les trois phases du scénario de mobilité. L'objectif principal des activités de PluriMobil est de promouvoir la réflexivité et l'autonomisation des apprentissages (voir Egli Cuenat et al., 2015 ; Egli Cuenat, 2018).

Suite à une commande du CELV, PluriMobil se base d'une part sur un usage ciblé d'instruments développés par le Conseil de l'Europe, notamment le Portfolio européen des langues ou l'Autobiographie des rencontres interculturelles (ARI, Conseil de l'Europe, 2009). D'autre part, il intègre d'autres sources pour la réflexion sur les identités multiples ou le plurilinguisme ${ }^{11}$ voire pour l'élaboration de mini-projets ethnographiques.

\footnotetext{
${ }^{11}$ Notamment les résultats du projet DYLAN, pour plus de détails voir Egli Cuenat \& Höchle Meier (2016).
} 
Le tableau 1 suivant montre de manière exemplaire comment les activités réflexives proposées dans les plans de leçons peuvent être agencées dans la séquentialité de la mobilité.

Tableau 1 - Mini-scénario exemplaire pour le secondaire supérieur en formation professionnelle.

\begin{tabular}{|c|c|}
\hline & $\begin{array}{l}\text { ACTIVITES ET EXPERIENCES à mettre en ouvre dans le cadre d'un séjour de mobilité de } 3 \\
\text { semaines pour des élèves du secondaire supérieur professionnel, basées sur un choix de plans } \\
\text { de leçons de PluriMobil : } \\
\text { - Plans de leçons No } 3,6 \text { et } 9 \text { pour le secondaire supérieur professionnel } \\
\text { - Plans de leçon No } 2 \text { pour le secondaire supérieur général }\end{array}$ \\
\hline 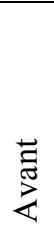 & $\begin{array}{l}\text { PL } 9 \text { et PL } 6 \text { : Evaluation de la compétence langagière et fixation de buts avec le Portfolio européen } \\
\text { des langues (PEL); préparation d'un journal d'apprentissage pour documenter les progrès } \\
\text { linguistiques } \\
\text { PL } 2 \text { : Réflexion sur sa propre identité multiple à l'aide d'un schéma (Paige et al., 2006) } \\
\text { PL } 3 \text { : Préparation à l'observation et à la documentation d'expériences avec l'Autobiographie des } \\
\text { rencontres interculturelles (ARI) }\end{array}$ \\
\hline & $\begin{array}{l}\text { PL } 9 \text { et PL } 6 \text { : Utilisation d'un journal d'apprentissage, observation sur la progression au niveau des } \\
\text { compétences langagières, réflexion sur les stratégies de communication } \\
\text { PL } 2 \text { : Mon identité multiple : réflexion individuelle } \\
\text { PL } 3 \text { : Documentation d'expériences et observations au moyen de carnets ou téléphones portables } \\
\text { d'expériences pour se préparer à compléter l'ARI }\end{array}$ \\
\hline 离 & $\begin{array}{l}\text { PL } 6 \text { et PL } 9 \text { : Présentation du journal d'apprentissage, réflexions sur les objectifs (en référence au } \\
\text { PEL) et sur le processus d'apprentissage linguistique } \\
\text { PL } 3 \text { : Réflexion sur une rencontre interculturelle particulière vécue durant la mobilité sur la base de } \\
\text { l'ARI et la documentation établie durant le séjour de mobilité } \\
\text { PL } 2 \text { : Retour sur le schéma de l'identité multiple, réflexion collective }\end{array}$ \\
\hline
\end{tabular}

La figure 2 ci-dessous, tirée du plan de leçons 6 de PluriMobil montre comment, à l'aide du Portfolio européen des langues (PEL), les étudiants sont encouragés à se fixer des objectifs d'apprentissage linguistique à partir d'une grille pour l'avant (anticiper, se préparer), le pendant (se donner les moyens pour contrôler les acquisitions in situ et pour s'insérer de façon adéquate dans de nouveaux groupes), l'après (vérifier si les acquisitions ont eu lieu).

Figure 2 - Extrait du plan de leçon 6 pour le secondaire supérieur professionnel, intitulé Le PEL Comment j'apprends et comment je veux apprendre? 


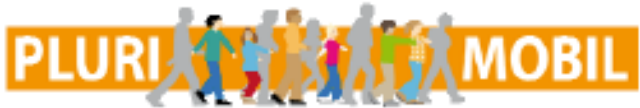

EXEMPLE 2

Objectifs pour le stage (CECR B1)

Grâce à cette liste, vous vous fixez des objectifs sur lesquels vous allez vous concentrer pendant le stage':

Avant le stage, listez des objectifs linguistiques que vous aimeriez atteindre. Pensez à ce que vous pouvez faire pour les atteindre.

Durant le stage, vérifiez que vous faites bien ce que vous aviez planifié.

Après le stage, indiquez les objectifs que vous avez de fait atteints.

\begin{tabular}{|l|l|l|l||}
\hline Objectifs & $\begin{array}{l}\text { AVANT } \\
\text { Que pouvez-vous faire pendant la } \\
\text { mobilité pour atteindre vos objectifs? } \\
\text { Expliquez. }\end{array}$ & $\begin{array}{l}\text { PENDANT } \\
\text { Qu'avez-vous fait réellement? } \\
\text { Expliquez. }\end{array}$ & $\begin{array}{l}\text { APRES } \\
\text { Avez-vous } \\
\text { atteint vos } \\
\text { objectifs? } \\
\text { Cochez, si } \\
\text { s'est le cas. }\end{array}$ \\
\hline $\begin{array}{l}\text { LIRE } \\
\text { Je sais comprendre des instructions } \\
\text { écrites, par exemple : dans un e- } \\
\text { mail. }\end{array}$ & & & \\
\hline $\begin{array}{l}\text { Je sais comprendre des instructions } \\
\text { dans un programme pour } \\
\text { l'ordinateur s'il y a aussi des } \\
\text { symboles ou des images. }\end{array}$ & & & \\
\hline $\begin{array}{l}\text { Je sais comprendre des posters / } \\
\text { des panneaux sur mon lieu de } \\
\text { travail. }\end{array}$ & & & \\
\hline $\begin{array}{l}\text { Je sais comprendre les comptes- } \\
\text { rendıc d'ıne réınion. }\end{array}$ & & & \\
\hline PARLER & & & \\
\hline $\begin{array}{l}\text { Je sais commander quelque chose à } \\
\text { manger ou à boire de manière } \\
\text { simple, par exemple : à la cantine. }\end{array}$ & & & \\
\hline $\begin{array}{l}\text { Je sais prendre part à une } \\
\text { conversation sur un sujet familier, } \\
\text { par exemple: comment j'ai passé } \\
\text { mon dimanche. }\end{array}$ & & & \\
\hline
\end{tabular}

Les étudiants sont également encouragés à réfléchir sur la connaissance de stratégies d'apprentissage avant le départ : c'est une phase de conscientisation qui vise l'autonomisation. Il s'agit donc d'un outil de médiation permettant à l'apprenant, quel qu'il soit, un début de gestion autonome de son processus d'acquisition langagière : dans la phase avant, les questions prévues facilitent la préparation langagière à la mobilité en référence à des situations professionnelles concrètes (situation de mobilité > processus de projection à favoriser) ; pendant la mobilité, l'apprenant réfléchit et travaille sur ses ressources langagières de façon à s'insérer de façon efficace dans les situations communicatives qu'il rencontre (entrée dans un nouveau groupe $>$ processus d'inclusion à encourager) et peut ajuster ses ressources à tout nouveau besoin qui se fera ressentir (perception de l'altérité > processus de focalisation à soutenir). Cet outil est destiné à enclencher des processus de réflexivité en sollicitant l'agentivité des apprenants en relation avec les compétences langagières, un des acquis principaux visés par les projets de mobilité à des fins d'apprentissage.

Figure 3 - Extrait du plan de leçon 2 pour le secondaire supérieur Mon identité multiple. 


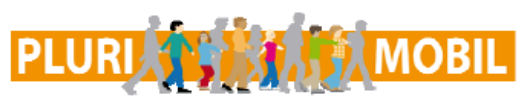

EXEMPLE 3

Dessinez : “Ma diversité culturelle”

Cette activité va vous aider à prendre conscience que vous êtes membre de différents groupes qui influencent votre identité culturelle.

Vous pouvez écrire, dans les cercles ci-dessus, des mots qui, selon vous, vous décrivent ou sont une partie importante de qui vous êtes et de comment vous vous identifiez par rapport aux autres (frère, étudiant, suisse, chrétien, démocrate, etc.).

Variante : Vous pouvez aussi mettre au centre le nom du pays où vous vous rendez pour la mobilité.

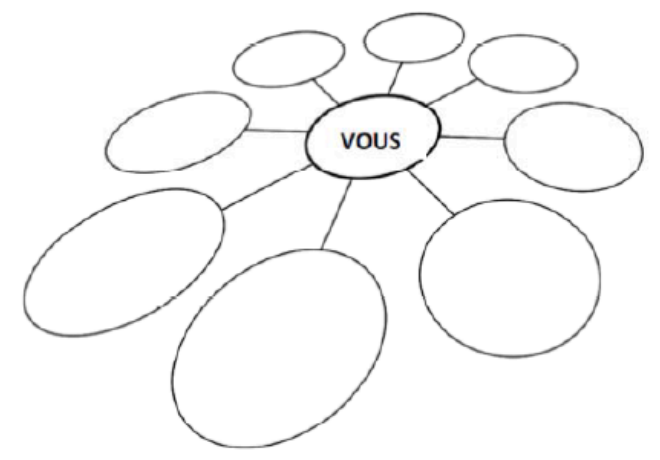

Voici quelques questions qui peuvent vous aider dans votre réflexion et vos choix :

Si vous deviez seulement choisir un cercle, lequel choisiriez-vous? Si vous deviez choisir deux cercles ? Pourquoi s'agit-il d'aspects importants de votre identité culturelle ?

Avez-vous eu des difficultés à compléter les huit cercles ?Y avait-il suffisamment de cercles ? Qu'avez-vous appris sur vous-même avec cet exercice ? Avez-vous trouvé un élément que les autres ne reconnaissent pas vraiment en vous? Est-ce ok pour vous ou est-ce frustrant ? [...]

Comment, pensez-vous, que ces cercles pourraient changer durant la mobilité ? Par exemple avez-vous indiqué votre nationalité dans l'un des cercles ? La classe sociale ? Les langues que vous parlez ? [...]

D’après: Paige, M. R./Cohen, A. D. et al. Maximizing Study Abroad. A Students' Guide to Strategies for Language and

La figure 3 se référant à une activité basée sur Paige, Cohen et al. (2006) veut sensibiliser les étudiants à leurs appartenances à des communautés multiples et l'éventuelle transformation à travers le processus de mobilité. Cet outil de médiation vise une réflexivité qui touche davantage des acquis profondément transformationnels, ce que nous appellerions des "dimensions sensibles » : les représentations de sa propre identité plurielle. La réflexion proposée se situe dans une perspective de préparation à la mobilité (situation de mobilité $>$ processus de projection à enclencher), d'intégration positive (participation à de nouveaux groupes $>$ processus d'inclusion à encourager) et d'ajustement / révision de ses propres représentations (perception de l'altérité $>$ processus de focalisation à faciliter).

La troisième activité préconisée dans le mini-scénario de mobilité, enfin, prévoit le travail avec l'Autobiographie des rencontres interculturelles (ARI, Conseil de l'Europe, 2009). Il s'agit d'un questionnaire permettant à l'apprenant de décrire et d'analyser la rencontre avec une autre personne qui l'a marqué, en focalisant, par exemple, sur la description précise des circonstances de la rencontre, ses propres sentiments et les sentiments de l'autre personne lors de la rencontre, les 
différences et les similitudes présupposées de la perception de la rencontre par les deux acteurs impliqués, les émotions déclenchées ou les conséquences de la rencontre. Le plan de leçon de PluriMobil prévoit la description très fine d'une rencontre passée avant la mobilité et une tâche de documentation et collection de matériaux (perception de l'altérité $>$ processus de focalisation $\&$ situation de mobilité $>$ processus de projection à soutenir), la rencontre consciente pendant la mobilité (entrée dans un groupe $>$ processus visant l'inclusion à cibler) et un retour conscient sur une rencontre faite dans le cadre de la mobilité (perception de l'altérité > processus de focalisation et d'ajustement à favoriser).

Les plans de leçons de PluriMobil intègrent différents instruments dont certains encore basés sur des approches différentialistes ou « solides » des cultures, c'est-à-dire des approches encourageant les apprenants à se concentrer sur les différences culturelles (plutôt que de mettre en valeur ce qui est commun) et à attribuer aux individus des identités statiques liées à des cultures ou des langues nationales (Dervin, 2016 : 74). Parmi ces outils, on peut nommer p.ex. Miroirs et fenêtres et en partie aussi l'Autobiographie des rencontres interculturelles (cf. les fiches exemples, p.4). D'autres instruments souscrivent clairement à une approche socio-constructiviste mettant en valeur les appartenances multiples et insistant à la fois sur les ressemblances et les différences, tenant compte du caractère «liquide», c'est-à-dire mouvant (Bauman, 2004, inter alia) ${ }^{12}$ des positionnements identitaires et de l'instabilité des discours culturels, corrélats nécessaires du mouvement de globalisation contemporain. Avec Dervin (2016, 79 inter alia), on peut qualifier cette approche qui tient à la fois du «solide» et du « liquide» comme janusienne, terme dérivé du dieu romain possédant deux têtes, l'une tournée vers le passé et l'autre vers l'avenir, gardien des portes, des fins et des commencements. Elle est liée au caractère éclectique du choix des instruments et à l'évolution des idées des auteurs de l'instrument PluriMobil durant la période d'élaboration; elle est également un miroir des contradictions inhérentes à l'interprétation, l'analyse et la construction des rencontres interculturelles (voir également Egli Cuenat, 2018 : 78).

Le manuel de PluriMobil (Egli Cuenat et al., 2015 : 15) met un accent fort sur la complexité des apprentissages et positionnements culturels, met en garde contre les dangers d'une focalisation exagérée sur les différences culturelles, l'essentialisme ou le culturalisme et fournit des orientations pour un usage sélectif et informé des plans de leçons par les enseignant-e-s (cf. également Egli Cuenat, 2018).

\section{Conclusions}

A la suite des réflexions et exemplifications jusqu'ici présentées, le modèle initialement proposé parait fonctionner comme un outil conceptuel apte à rendre compte des processus transformationnels qu'un projet de mobilité à des fins d'apprentissage déclenche. En même temps, il justifie et confirme l'utilité de différentes formes, approches, activités et outils de médiation que des dispositifs voire des acteurs de la médiation (institutionnels ou non) peuvent offrir à des individus en mobilité. Le modèle conceptuel général aide ainsi à comprendre le fonctionnement d'un contexte d'apprentissage particulier (la mobilité à des fins d'apprentissage) et la fonction majeure que la médiation - aussi bien cognitive que relationnelle - en passant par des activités langagières de médiation diverses peut avoir pour une véritable mobilité symbolique.

De ces réflexions et exemplifications découle la nécessité de former les enseignants/les formateurs comme médiateurs des processus de mobilité et de leur donner des outils abordables et facilement

\footnotetext{
${ }^{12}$ A remarquer qu'en utilisant les termes de liquide et solide, empruntés à Z. Bauman (2004), Dervin (2016 inter alia) met en avant le caractère nécessairement mouvant et instable de toute définition identitaire et non pas les angoisses potentiellement engendrées par la perte de stabilité par le mouvement de la globalisation décrites par Bauman.
} 
accessibles pour agir en tant que tels, tout en respectant les impératifs de la pratique du quotidien. PluriMobil, l'outil d'encadrement pédagogique de la mobilité à des fin d'apprentissage exposé de manière plus détaillée ici, apparait, selon le modèle conceptuel présenté, comme un dispositif de médiation mis à la disposition par une institution internationale (le CELV) à d'autres institutions, par exemple, dans le cas de la formation initiale des enseignants, des instituts universitaires de formation européens, mais aussi, dans le cas des élèves des différents niveaux scolaires, d'institutions plus locales - des écoles voire des professionnels individuels (formateurs de futurs enseignants ou enseignants). Ces institutions et ces professionnels sont envisagés comme des intermédiaires devant agir sur les tensions possibles (de signe aussi bien positif que négatif) entre des acteurs placés dans des situations de mobilité et les nouvelles expériences, vécus, ressentis, représentations, connaissances, personnes etc. qu'ils sont amenés à rencontrer. La finalité étant d'aider ces acteurs à "dénouer» positivement ces tensions, en faveur de leur développement personnel et professionnel.

Qu'il nous soit permis de conclure cet article en soulignant que l'optique générale adoptée dans notre texte est aussi celle de la médiation entre la production théorique ou scientifique et le terrain de l'enseignement des langues que nous percevons comme deux pôles en tension, qu'il s'agit d'articuler non pas hiérarchiquement (que ce soit dans un sens ou dans un autre), mais en complémentarité, en réciprocité, sur un plan d'égalité. La visée de ce processus se construisant dans un mouvement alterné, récursif et ascensionnel est l'enrichissement réciproque de la théorie et de la pratique se fondant sur leur consubstantialité ${ }^{13}$.

La référence aux travaux du Conseil de l'Europe, enfin, ne dérive pas uniquement du fait que nous avons travaillé à quelques-unes des actions et projets de cette institution, mais en tant que ces travaux représentent à nos yeux un levier puissant pour faire passer des concepts - élaborés dans les domaines de la recherche et de la théorie - dans la pratique de l'enseignement.

\section{Bibliographie}

Bange, P. (1992). À propos de la communication et de l'apprentissage de la L2, notamment dans ses formes institutionnelles. Acquisition et interaction en langue étrangère (AILE, 1), 53-85.

Bauman, Z. (2004). Identity. Cambridge : Polity Press.

Blanchet, P. \& Chardenet, P. (dir.). (2011). Guide pour la recherche en didactique des langues et des cultures ( $2^{\mathrm{e}}$ édition). Paris : Editions des archives contemporaines.

Bruner, J. (1978). The role of dialogue in language acquisition. Dans A. Sinclair, R. Jarvella and W. J. M. Levelt (dir.), The Child's Conception of Language (p. 241-256). New York : Springer-Verlag. Byram, M., Gribkova, B. \& Starkey, H. (2002). Developing the Intercultural Dimension in Language Teaching: A Practical Introduction for Teachers. Strasbourg : Conseil de l'Europe.

Commission Européenne (2009). Livre vert-promouvoir la mobilité à des fins d'apprentissages. Bruxelles : Commission des communautés européennes.

Conseil de l'Europe (2001). Cadre européen commun de référence pour les langues : apprendre, enseigner, évaluer. Paris : Didier.

Conseil de 1'Europe (2009). Autobiographie des rencontres interculturelles. Strasbourg: Unité des Politiques Linguistiques. Repéré à https://www.coe.int/fr/web/autobiography-interculturalencounters/home (consulté le 06/09/19).

\footnotetext{
${ }^{13}$ En ce sens nous partageons pleinement une citation de Feuerstein, reprise par Lantolf \& Poehner (2014 : 160) : "Notre position fondamentale est que nous avons besoin à la fois de théorie et de pratique. La façon dont nous développons des concepts guide nos pratiques, et ce que nous faisons (nos pratiques) contribue à façonner notre théorie". (Feuerstein et al., $2010: 5$, notre traduction).
} 
Conseil de l'Europe (2018). Cadre européen commun de référence pour les langues : apprendre, enseigner, évaluer - Volume complémentaire avec de nouveaux descripteurs. Strasbourg : Conseil de l'Europe.

Coste, D. (2019). Savoir prendre ses distances. Dans Causa, M. \& Stratilaki, S. (dir.), Distance(s) et didactique des langues - L'exemple de l'enseignement bilingue (p. 27-53). Bruxelles : E.M.E. éditions.

Coste, D. \& Cavalli, M. (sous presse). Des traits constitutifs de toute médiation ?. Dans M. Di Gioa et M. Marcon (dir.), L'essentiel de la médiation. Le regard des sciences humaines et sociales. Berne : Maison d'édition P.I.E. Peter Lang SA, Éditions Scientifiques Internationales.

Coste, D. \& Cavalli, M. (2019). Migration et politiques linguistiques éducatives - Le rôle majeur de la médiation. European Journal of Language Policy, 10(2), Liverpool : University Press.165-186.

Coste, D. \& Cavalli, M. (2018). Altérité, communauté, médiation, mobilité : des notions à manipuler avec précaution? Dans C. Jeoffrion et M.-F. Narcy-Combes (dir.), Perspectives plurilingues en éducation et formation (p. 243-256). Des représentations aux dispositifs. Rennes : Presses Universitaires de Rennes.

Coste, D. \& Cavalli, M. (2015). Éducation, mobilité, altérité. Les fonctions de médiation de l'école. Strasbourg : Conseil de l'Europe. Repéré à http://www.coe.int (consulté le 06/09/19).

Coste, D. \& Cavalli, M. (2014a). Réexaminer et enrichir le dispositif conceptuel du CECR Altérité, mobilité, communauté à l'épreuve de la médiation. Strasbourg: Conseil de l'Europe (non publié).

Coste, D. \& Cavalli, M. (2014b). Extension du domaine de la médiation. Lingue Culture Mediazioni / Languages Cultures Mediation, 1(1-2): 101-117. Repéré à https://www.ledonline.it/index.php/LCM-Journal/article/view/750 (consulté le 06/09/19)

Coste, D., Moore, D. \& Zarate, G. (2009). Compétence plurilingue et pluriculturelle (Version révisée et enrichie d'un avant-propos et d'une bibliographie complémentaire). Strasbourg : Conseil de l'Europe ( $1^{\text {ère }}$ éd. 1997).

Dervin, F. (2016). Interculturality in Education: A Theoretical and Methodological Toolbox. London : Palgrave.

Egli Cuenat, M. (2018). PluriMobil - Pragmatic enhancement of intercultural learning before, during and after study abroad. Dans J. Jackson, S. Oguro (dir.), Intercultural interventions in study abroad (p. 175-189). Abingdon, Oxon, New York : Routledge.

Egli Cuenat, M., Brogan, K., Czura, A., Muller, C., Cole, J., Szczepańska, A., Bleichenbacher, L., Höchle Meier, K. \& Wolfer, B. (2015). PluriMobil: Plurilingual and intercultural learning through mobility: Practical resources for teachers and teacher trainers. Graz : Council of Europe.

Egli Cuenat, M. \& Höchle Meier, K. (2016). PluriMobil meets DYLAN - practical resources for supporting intercultural and plurilingual learning in vocational student mobility. Dans G. Lüdi, K. Höchle Meier, P. Yanaprasart (dir.), Managing plurilingual and intercultural practices in the workplace (p. 294-312). Amsterdam : John Benjamins.

Ehrenreich, S, Woodman, G. \& Perrefort, M. (dir.) (2008). Auslandsaufenthalte in Schule und Studium: Bestandsaufnahmen aus Forschung und Praxis. Münster: Waxmann.

Fairclough, N. (éd.) (1992). Critical Language Awareness. Harlow : Longman.

Fairclough, N. (2001). Language and Power ( $2^{\mathrm{e}}$ édition). London : Routledge.

Gohard-Radenkovic, A. (2007). Comment analyser les rapports identitaires entre groupes et entre individus en situation de mobilité ? Igitur, Lingue / Culture / Identità, Rivista Annuale di Lingue, Letterature et Culture Moderne, Anno VIII, Roma, Nuova Arnica Editrice, 41-56.

Guilherme, M. (2012). Critical language and Intercultural Communication Pedagogy. Dans Jackson, J. (dir.), The Routledge Handbook of Language and Intercultural Communication (p. 357 -371). London, New York : Routlege.

Guillaume-Hofnung, M. (2015). La mediation (6 $6^{\mathrm{e}}$ éd). Paris : PUF (Que sais-je ?) (1 ${ }^{\text {ère }}$ éd. 1995). 
Huver, E. \& Lorilleux, J. (dir.). (2018). Quelles médiations en didactique des langues et des cultures ? - Questions notionnelles et formatives. Recherches en didactique des langues et des cultures, 15(2) (https://journals.openedition.org/rdlc/2962, consulté le 12/09/19).

Jackson, J. (dir.). (2012). The Routledge Handbook of Language and Intercultural Communication. London : Routledge.

Jackson, J., \& Oguro, S. (dir.). (2018). Intercultural Interventions in Study Abroad. London: Routledge.

Kolb, D. A. (1984). Experiential learning: Experience as the source of learning and development (vol. 1). Englewood Cliffs, NJ: Prentice-Hall.

Kötter, M. (2013). Work experience as an opportunity for language learning. Dans J. Rymarczik (dir.). Foreign Language Learning Outside School: Places to See, Learn and Enjoy. Inquiries in Language Learning (vol.8, 17-34). Frankfurt am Main : New York: Peter Lang Edition.

Kramsch, C. (2009). The Multilingual Subject. Oxford: Oxford University Press.

Lantolf, J. P. \& Poehner, M. E. (2014). Sociocultural Theory and the Pedagogical Imperative in L2 Education: Vygotskian Praxis and the Research/Practice Divide. New York, London: Routledge.

Lave, J. \& Wenger, E. (1991). Situated Learning: Legitimate Peripheral Participation. Cambridge: Cambridge University Press.

Levy, D. \& Zarate, G. (dir.). (2003). La médiation et la didactique des langues et des cultures. Le Français dans le Monde. Recherche et applications. Paris : Clé international.

Lorilleux, J. \& Huver, E. (dir.). (2018). Quelles médiations en didactique des langues et des cultures ? - Démarches et dispositifs. Recherches en didactique des langues et des cultures, 15(3) (http://journals.openedition.org/rdlc/3224, consulté le 08/09/19).

Little, P. D., Dam, L. \& Legenhausen, L. (2017). Language Learner Autonomy: Theory, Practice and Research. Bristol : Multilingual Matters.

Molinié, M. (dir.). (2006). Biographie langagière et apprentissage plurilingue. Le français dans le monde, 39(janvier).

Molinié, M. (dir.). (2014). (Se) représenter les mobilités : dynamiques plurilingues et relations altéritaires dans les espaces mondialisés. GLOTTOPOL, 24(juillet).

Murphy-Lejeune, E. (2004). L'Etudiant européen voyageur, un nouvel étranger. Paris : DidierCrédif.

Paige, M. R., Cohen, A. D., Shively, R. L., Emert, H. A. \& Hoff J.G. (2006). Maximizing Study Abroad. A Students' Guide to Strategies for Language and Culture Learning and Use. Minneapolis : Board of Regents University of Minnesota.

Paige, R.M. \& Vande Berg, M. (2012). Why students are and are not learning abroad: A review of recent research. Dans M. Vande Berg, R.M. Paige, and K.H. Lou (dir.), Student Learning Abroad: What Our Students Are Learning, What They're Not, and What We Can Do about It (29-58). Sterling, VA : Stylus.

Roussel, S. \& Gaonac'h, D. (2017). L'apprentissage des langues. Paris : Retz.

Six, J.-F. (1990). Le temps des médiateurs. Paris : Le Seuil.

Stathopoulou, M. (2015). Cross-Language Mediation in Foreign Language Teaching and Testing. Bristol, Buffalo, Toronto : Multilingual Matters.

Vygotsky, L.S. (1992). Pensiero e linguaggio. Bari : Laterza (éd. or. 1934).

Zarate, G., Gohard-Radenkovic, A., Lussier, D. \& Penz, H. (2003). Médiation culturelle et didactique des langues. Graz : CELV /ECML. 